\title{
Urchristliche Missionswege nach Rom: Haushalte paganer Herrschaft als jüdisch-christliche Keimzellen
}

\author{
von Peter Lampe \\ (Universität Heidelberg, Kisselgasse 1, D-69117 Heidelberg)
}

Für Prof. em. Robert Jewett

In den 40 er Jahren des ersten Jahrhunderts fasste in Rom das Christentum zuerst in einer oder mehreren Synagogen Fuß. Die christliche Botschaft entfachte Unruhe unter den stadtrömischen Juden, bis Claudius die herausragenden Köpfe dieser innerjüdischen Auseinandersetzung der Stadt verwies. ${ }^{1}$

Bislang ungelöst ist, auf welchen Wegen das Christentum die römischen Synagogen erreichte. Durch das Zusammensetzen von Mosaiksteinen verschiedenster Quellen deutet sich ein Lösungsmodell an.

1.1. Der erste Clemensbrief $(63,3 ; 65,1)$ nennt einen römischen Christen namens Valerius Biton, der in den 90 er Jahren des 1 . Jahrhunderts n. Chr. als alter Mann der römischen Delegation angehörte, die den ersten Clemensbrief nach Korinth überbrachte und der Briefbotschaft dort Nachdruck verlieh. Als alter Mann in den 90 er Jahren des 1. Jahrhunderts dürfte Valerius Biton in den 30 er oder 40 er Jahren geboren worden sein. Die Anfänge des Christentums in Rom erlebte er als Kind; von Jugend auf gehörte er zum stadtrömischen Christentum. Sein Name identifiziert ihn als freigelassenen Sklaven der gens Valeria oder als Nachkomme eines solchen Freigelassenen.

1.2. Den zweiten Mosaikstein liefert ein paganes lateinisches Epigraph aus Rom aus dem 1. Jahrhundert n. Chr. (CIL VI 27948). Die Grabinschrift listet Freigelassene der gens Valeria auf, darunter eine Valeria Maria. »Maria« stellt in diesem Fall deutlich nicht ein Gentilicium, sondern ein Cognomen dar, was in CIL VI nur selten begegnet. Üblicherweise bezeichnet »Maria« - wie »Marius« - in den stadtrömischen lateinischen Inschriften die gens Maria. Nur siebenmal läßt sich das Cognomen »Maria« in CIL VI greifen. ${ }^{2}$ Das Cognomen jedoch repräsentiert den semitischen Namen, so daß unsere Valeria Maria des 1. Jahrhunderts n. Chr. aller Wahrscheinlichkeit Jüdin oder Judenchristin war.

1 Sueton, Claud. 25,4; Apg 18,2; Orosius, Hist. 7,6,15 f.; vgl. Cassius Dio 60,6,6f.; dazu z. B. P. Lampe, Die stadtrömischen Christen in den ersten beiden Jahrhunderten. Untersuchungen zur Sozialgeschichte (WUNT 2/18), Tübingen ${ }^{2} 1989,4-8$. Die Diskussion über das »Claudiusedikt « braucht hier nicht erneut aufgerollt zu werden.

2 CIL VI 14025; 27948; 12907; wohl auch 11175; 19039; 13717; 10881. Das Gentilicium »Maria« begegnet in CIL VI dagegen ca. 108 mal. 
Valeria Maria wird in der Inschrift als eine der Freigelassenen eines Lucius Valerius Diogenes ausgewiesen. ${ }^{3}$ Diogenes hatte einen Lucius Valerius Hiero beerbt, Hiero seinerseits einen Lucius Valerius Papia. Starb Valeria Maria spätestens in den 90 er Jahren des 1. Jahrhunderts n. Chr., so lassen sich für die Todesdaten des Diogenes, des Hiero und des Papia spätestens die 80 er, 50 er beziehungsweise 20 er Jahre supponieren. Das heißt, Lucius Valerius Papia wurde in jedem Fall im 1. Jahrhundert v. Chr. geboren.

Einen judenfreundlichen Senator Lucius Valerius des 1. Jahrhunderts v. Chr. nennt Josephus, Ant. 14,145: Im Jahre 47 v. Chr. brachte ein praetor urbanus Lucius Valerius, Sohn eines Lucius Valerius, in eine von ihm geleitete Senatssitzung den Antrag ein, die Freundschaft und Verbrüderung mit dem jüdischen Volk erneut zum Ausdruck zu bringen und an »die freien Städte und Könige« des römischen Einflußbereichs Briefe zu senden, die den Juden unbehelligten Zugang zu den Häfen und Gebieten dieser Briefadressaten sicherten. Lucius Valerius hatte vorher eine dreiköpfige jüdische Gesandtschaft angehört, die um Entsprechendes gebeten hatte. Der Senat stimmte dem Antrag des Lucius Valerius zu. ${ }^{4}$

Diesen judenfreundlichen Lucius Valerius mit der familia der jüdischen Maria, der Freigelassenen eines Lucius Valerius Diogenes, in Verbindung zu bringen, liegt nahe, auch wenn der letzte Beweis nur durch neues Inschriftenmaterial erbracht werden könnte.

1.3. Als möglicher Patron valerischer jüdischer Freigelassener kommt auch der römische Rhetor Marcus Valerius Messalla Corvinus in Frage. Er war als Konsulssohn um 64 v. Chr. geboren worden. Nach Aufenthalten in Griechenland, Makedonien, Kleinasien und

3 Das Epigraph präsentiert zwei Gruppen von Freigelassenen, denen von ihren Patronen ein Grabplatz überlassen worden war. Diese beiden Patrone, Lucius Valerius Amphion und Lucius Valerius Diogenes, hatten den Platz ihrerseits von demselben Erblasser (wohl von ihrem Vater) übernommen: von einem Lucius Valerius Hiero. - Amphion wird an erster Stelle vor Diogenes genannt. Deshalb ist sinnvoll anzunehmen, daß die erste Freigelassenen-Gruppe von Amphion abhing, während die zweite, zu der unsere Maria zählte, sich auf Diogenes bezog. In der zweiten Gruppe trägt einer der Freigelassenen bezeichnenderweise wiederum den Namen Diogenes.

4 Dieses Senatus-Consultum mit 1Makk 15,15-24; 12,16-17; 14,22-24 zu verbinden und dann entgegen Josephus in die 130 er Jahre v. Chr. zu datieren (für viele, z. B. T. R. S. Broughton, The Magistrates of the Roman Republic I, New York 1951, 491-492), ist möglich - und der gelehrte Streit entsprechend. Beiden Texten zufolge wurde von der jüdischen Gesandtschaft ein goldenes Schild dargebracht. Beide Male wurden Freundschaft erneuert und Schutzbriefe ausgestellt. Beide Male zählte ein Numenius, Sohn des Antiochus, zur jüdischen Delegation. Beide Male führte ein Senator Lucius die Feder. In beiden Fällen trat ein »Sohn des Jason« als zweiter Gesandter auf, nur hieß er im 1Makk Antipater, während Josephus ihn Alexander nennt. (Abweichend von 1Makk kennt Josephus darüber hinaus einen dritten Delegierten namentlich.) Falls Josephus' Senatus-Consultum dem Kontext von 1Makk 15 entsprechend tatsächlich in die 130 er Jahre v. Chr. gehörte, Josephus sich also in der zeitlichen Einordnung seiner (von 1Makk literarisch unabhängigen) Quelle geirrt hätte, so war der federführende Senator Lucius Valerius mit dem Konsul von 131 v. Chr., Lucius Valerius Flaccus, identisch, der als flamen Martialis das Konsulat erreichte und wohl zum Kreis um die Gracchen zählte (vgl. M. DeißmannMerten, Art. Valerius. 30. Lucius Valerius Flaccus, KP 5 [1975] 1103). Unser judenfreundlicher Lucius Valerius gehörte dann nicht erst dem 1. Jahrhundert v. Chr., sondern bereits der zweiten Hälfte des 2. Jahrhunderts v. Chr. an. 
Alexandria $^{5}$ legte er im Jahre 40 v. Chr. in Rom ein gutes Wort für Herodes den Großen ein. ${ }^{6}$ Um 28 v. Chr. trat er die Statthalterschaft in Syrien an, wo er vor Antiochia eine Gladiatorenbande auflöste. ${ }^{7}$

2. Valerius Messalla erhielt zusammen mit Marcus Agrippa im Jahre 29 v. Chr. ehrenhalber das Haus des M. Antonius auf dem Palatin. ${ }^{8}$ Marcus Agrippa begab sich gegen Ende der 20 er Jahre v. Chr. als Generalstatthalter in die östlichen Provinzen; im Jahre 17 v. Chr. machte er sich erneut in den Osten auf. Mit Herodes dem Großen unterhielt er ab 15 v. Chr. eine enge Freundschaft. Josephus bescheinigt ihm freundliche Gesinnung gegenüber den Juden. ${ }^{9}$

Zu Agrippas stadtrömischem Haushalt zählten jüdische Sklaven und Freigelassene, die im 1. Jahrhundert n. Chr. eine epigraphisch belegte $\sigma u v \alpha \gamma \omega \gamma \dot{\eta}$ 'A $\gamma p ı \pi \pi \eta \sigma i \omega v$ bildeten. ${ }^{10}$ Ein anderer Patron dieser jüdischen synagogalen Gruppe als Marcus Agrippa selber kommt nicht in Betracht, es sei denn, man greift auf den in Rom aufgewachsenen Agrippa I oder gar dessen Sohn Agrippa II aus dem Hause Herodes' des Großen zurück.

3. »Volumnius« war das nomen gentile eines römischen Legaten, der im Jahre $8 \mathrm{v}$. Chr. in Syrien residierte und dessen Freundschaft sich Herodes der Große erfreute. ${ }^{11}$ Dieser Volumnius kommt am ehesten als Patron der jüdischen Freigelassenen und Sklaven in Frage, die die stadtrömische Synagoge der »Volumnenses« (1. Jahrhundert n. Chr.) gründeten. ${ }^{12}$

Fazit: Verschiedene römische Patrone lassen sich benennen, die im syrischen Osten des Reiches sich aufgehalten hatten, freundliche Beziehungen mit Herodes dem Großen unterhielten und in Rom jüdische Freigelassene und Sklaven zu ihrem Hausstand zählten. Diese liberti und servi bildeten - zumindest in zwei noch erkennbaren Fällen - je eigene synagogale Gemeinschaften und scheuten sich nicht, ihre Synagogen nach ihrem paganen Patron

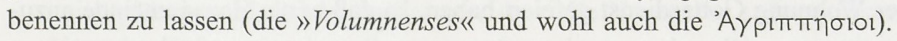

4. Daß sich schließlich auch eine stadtrömische Synagoge $(H) P O \triangle I \Omega N$ inschriftlich belegen läßt (CIJ I 173), verwundert nach allem nicht. Auch wenn diese Inschrift nicht ins 1. Jahrhundert n. Chr. gehört, kann - aufgrund des Namens - eine Entstehung dieser jüdischen Gemeinde in augusteischer Zeit nicht ausgeschlossen werden. Es besteht so eine weitere Möglichkeit, daß jüdische Freigelassene und Sklaven des herodianischen Königshauses in Rom eine eigene Synagoge unterhielten.

5. Im 1. Jahrhundert n. Chr. bildeten in Rom darüber hinaus Freigelassene des kaiserli-



5 Vgl. Cicero, Att. 12,32,2; Appian, Bell.civ. 4,159 f.; Cassius Dio 47,33,1 f.; Plutarch, Brut. 40.41; Velleius Paterculus 2,71,1.

6 Josephus, Ant. 14,384; Bell. 1,284; vgl. auch 1,243.

7 Cassius Dio 51,7,7; Tibull 1,3,1.

8 Cassius Dio 53,27,5.

9 Josephus, Ant. 15,350 f.; 16,12-16.21-62.

0 CIG 9907; CIJ I 503; 425; 365.

11 Josephus, Bell. 1,535 f. 538. 542; Ant. 16, 277 ff.332.335 ff.351.354.

2 CIL VI 29756; CIJ I 343; 402; 417; 523.

13 CIL VI 29757; CIG 9.902 f.; CIJ I 284; 301; 338; 368; 416; 496. Christen innerhalb des kaiserlichen Haushaltes lassen sich für die Mitte des 1. Jahrhunderts wenigstens für den Osten nachweisen: Phil 4,22. 
Daneben existierte zur selben Zeit schließlich die stadtrömische Synagoge der »Vernaculi ${ }^{14}$, deren Name jedoch nicht eindeutig interpretiert werden kann: Entweder assoziierten sich hier sogenannte Haussklaven, im Hause geborene Sklaven, oder aber autochthon römische Juden (im Gegensatz zu zugewanderten) fanden in dieser Synagoge sich zusammen.

6. Nicht nur jüdische Freigelassene und Sklaven einer paganen Herrschaft konnten je eigene Synagogen bilden, auch christliche liberti und/oder servi nicht-christlicher Herren fanden sich zu je eigenen christlichen Hausgemeinden zusammen. Paulus läßt in Röm 16,10-11 neben den anderen Gemeindekreisen in Rom

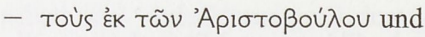

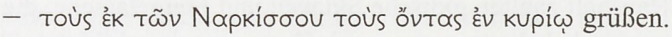

Daneben sandte er Grüße

- an die Hausgemeinde von Priska und Aquila,

- an die Christen, die mit Asynkritus, Phlegon, Hermes, Patrobas und Hermas zusammen waren, und

- an die Heiligen, die sich um Philologus, Julia, Nereus, dessen Schwester und Olympas scharten.

Gehen wir davon aus, daß die anderen 14 gegrüßten Personen des Kapitels zu keinem dieser fünf Kristallisationspunkte zählten und sie auch kaum nur einem einzigen weiteren Kreis angehörten, ergeben sich für die 50 er Jahre des 1. Jahrhunderts mindestens sieben gesonderte christliche Inseln in der Welthauptstadt. Anzeichen für ein räumliches Zentrum der verschiedenen über die Stadt verstreuten Christenkreise finden sich weder in dieser Zeit noch später in den ersten beiden Jahrhunderten an irgend einer Stelle. ${ }^{15}$ Jeder Kreis dürfte je für sich in einer Wohnung Gottesdienst gefeiert haben, so daß er als Hausgemeinde anzusprechen ist.

Für die beiden christlichen Zirkel in den Hausständen des Aristobul und des Narcissus ist deutlich, daß (a) die beiden Patrone keine Christen waren, sonst wären sie mitgegrüßt worden, und daß (b) nicht alle Freigelassenen und/oder Sklaven der beiden Haushalte zum stadtrömischen Christentum gehörten, sonst hätte Paulus viel einfacher formulieren können und schlicht »die Leute des Aristobul« und »die des Narcissus« grüßen lassen.

Aristobuls Name begegnet nur selten in Rom. ${ }^{16}$ Aristobul wird aus dem Osten nach Rom eingewandert sein (zusammen mit seinen christlichen Domestiken?). Möglich ist auch, daß er selber im Osten residierte und nur ein Teil seines Haushaltes in Rom etabliert war. $\mathrm{Da}$ der Name »Aristobul« in der herodianischen Familie beliebt war, paßt zu den obigen Daten, legt aber kein ausreichendes Fundament für weiterreichende Schlüsse.

7. Nicht nur jüdische oder christliche Sklaven und/oder Freigelassene eines Haushaltes bildeten selbständige religiöse Gemeinschaften. Auch die pagane Dienerschar eines Haushaltes konnte als selbständige kultische Einheit auftreten. In paganen Häusern und Besitzungen besorgten Sklavenkollegien den Larenkult oder den Genienkult des Herrn. Die Sklaven selber übten in diesen Kultgemeinschaften die Ehrenämter aus, auch die priesterlichen. ${ }^{17}$ Tacitus ${ }^{18}$

14 CIJ I 318; 383; 398; 494.

15 Vgl. Lampe, Christen (s. Anm. 1), 301-345.

16 Die maskuline Form nur zweimal in CIL VI: 17577; 29104.

17 Vgl. dazu Lampe, Christen (s. Anm. 1), 319 (Lit.).

18 Ann. 14,44,3. 
kennt römische Häuser, in denen das Gesinde eine Religiosität praktizierte, die den Hausherrn fremd war. Zu Beginn des 4. Jahrhunderts schließlich verehrten pagane Sklaven dann auch in Häusern christlicher Herren ihre Götterbilder. ${ }^{19}$

Das zusammengestellte Material macht deutlich:

a) Synagogale und christliche Hausgemeinden konnten sich bisweilen mit den Grenzen des Haushalts definieren, innerhalb derer ihre Mitglieder als Sklaven oder Freigelassene lebten und wirkten. Primärer Arbeits- und Lebenskreis und religiöser Gemeindezirkel verhielten sich wie zwei konzentrische Kreise zueinander.

b) Der Patron oder die Patronin des betreffenden Hausstands praktizierte selber eine andere Religiosität. ${ }^{20}$ Die synagogalen und christlichen Hausgemeinden organisierten sich in diesen Fällen aufgrund eigener Initiative, wurden aber von der Herrschaft geduldet.

c) Das fragmentarische Material benennt einige Besitzer und Patrone stadtrömischer jüdischchristlicher Sklaven und Freigelassener. Bisweilen hatten diese Patrone im syrischen Osten für eine Zeit gewirkt und Beziehungen mit Herodes gepflegt (Marcus Agrippa, Volumnius, vgl. Valerius). Affinität zum herodianischen Königshaus erschien in mindestens zwei Fällen wenigstens möglich (die Herodioi und die Aristobul-Leute).

d) Ein Nebeneinander von jüdischen und christlichen Sklaven und Freigelassenen läßt sich wenigstens in der gens Valeria (s. o. 1) und im kaiserlichen Haushalt (s. o. 5) nachweisen. Die Wahrscheinlichkeit ist groß, daß der am Ende des 1. Jahrhunderts n. Chr. bereits gealterte Christ Valerius Biton (1Clem 63,3; 65,1), der von Jugend auf zum stadtrömischen Christentum gehörte, durch valerische jüdische Freigelassene mit der christlichen Verkündigung in Kontakt gekommen war, vielleicht sogar die Jüdin oder Judenchristin Valeria Maria seine Mutter oder eine andere nahe Verwandte war. Doch bedürfte es neuen epigraphischen Materials, um letztere spezielle Hypothese substantiieren zu können. Mit Vorsicht dürfte immerhin allgemeiner formuliert werden: Die genannten paganen stadtrömischen Haushalte (in den gentes Valeria und Volumnia; des Agrippa; möglicherweise auch des Kaisers selber), das heißt, ihre jüdischen servi und liberti und deren Nachkommen, bieten sich (neben herodianischen Domestiken) als die Brückenköpfe an, über die das Judenchristentum in den 40 er Jahren des 1 . Jahrhunderts n. Chr. aus dem syro-palästinischen Osten in die Welthauptstadt Rom vordrang.

19 Can. 41 Elvira. - Als paganen Sklaven in christlichem Hause vgl. im 1. Jahrhundert Onesimus, der bis zu seiner Bekehrung durch Paulus heidnischer Religiosität anhing (Phlm). Freilich ist nicht erkennbar, in welcher Form er letztere praktizierte, ob zusammen mit anderen paganen Sklaven im Hause des Philemon oder außerhalb. Zu christlichen Sklaven in paganen Häusern vgl. auch 1Tim 6,1; Tit 2,9-10; Origenes, c.Cels. 3,55 .

20 Auf alle namentlich genannten Haushalte trifft dies zu. Lediglich die Herodioi würden eine Ausnahme bilden, ebenfalls die Agrippa-Domestiken, falls ihr Patron wirklich Agrippa I. oder Agrippa II. gewesen wäre. 